\title{
Role of the mTOR pathway in minor salivary gland changes in Sjogren's syndrome and systemic sclerosis
}

\author{
Zeki Soypaçacı ${ }^{1 *}$, Zeynep Zehra Gümüş ${ }^{2}$, Fulya Çakaloğlu ${ }^{3}$, Mustafa Özmen ${ }^{4}$, Dilek Solmaz ${ }^{4}$, Sercan Gücenmez ${ }^{4}$, \\ Önay Gercik ${ }^{4}$ and Servet Akar $^{4}$
}

\begin{abstract}
Background: To examine the activity of the mammalian target of rapamycin (mTOR) pathway and its regulators, transforming growth factor (TGF)- $\beta 1$ and phosphatase and tensin homolog (PTEN), in minor salivary gland biopsies of Sjogren's syndrome (SS) and systemic sclerosis (SSC) patients.

Methods: We retrospectively evaluated SS, SSC, and SS-SSc overlap patients admitted to our outpatient rheumatology clinic between January 2007 and December 2015 who underwent a minor salivary gland biopsy. Patient demographics and some clinical features were obtained from hospital records. Immunohistochemistry was used to analyze total mTOR, total PTEN, and TGF- $\beta 1$ expression in the biopsied tissues. The biopsy specimens were also examined for the presence and degree of fibrosis.

Results: Minor salivary gland biopsies of 58 SS, 14 SSc, and 23 SS-SSc overlap patients were included in the study. There was no significant difference in mTOR expression between these groups $(P=0.622)$. PTEN protein was expressed in $87.2 \%$ of patients with SS, $57.9 \%$ with overlap syndrome, and $100 \%$ of the SSC patients, and these differences were statistically different $(P=0.023)$. Although ductal epithelial TGF- $\beta 1$ expression was similar between the groups $(P=0.345)$, acinar cell expression was found to be more frequent in the SSC (72.7\%) and overlap patients (85.7\%) in comparison with the SS cases (58.2\%; $P=0.004)$.

Conclusion: mTOR may be one of the common pathways in the pathology of both SS and SSc. Hence, there may be a role for mTOR inhibitors in the treatment of both diseases. Additionally, PTEN and TGF- $\beta 1$ expression may be a distinctive feature of SSC.
\end{abstract}

Keywords: Target of rapamycin proteins, mTOR pathway, Sjogren's syndrome, Systemic sclerosis, PTEN protein, Human, Transforming growth factor beta

\section{Background}

Sjogren's syndrome (SS) is a chronic, systemic, inflammatory disease [1]. The characteristic pathologic findings for this disorder are lymphocytic infiltration of the exocrine glands leading to autoantibody production and tissue destruction $[1,2]$. Consistent with its pathogenesis, the first symptoms of SS are generally xerostomia and keratoconjunctivitis sicca [2]. SS can occur as an isolated primary condition or secondary to another connective

\footnotetext{
* Correspondence: soypacaci@yahoo.com

${ }^{1}$ Department of Internal Medicine, Division of Nephrology, Izmir Katip Celebi

University School of Medicine, Karabağlar, 35360 Izmir, Turkey

Full list of author information is available at the end of the article
}

tissue disease. At the beginning of SS onset, CD4-positive $\mathrm{T}$ helper cells play a pathogenic role whereas, in late-term SS, B cells play a predominant role. Recent studies have indicated that epithelial cells are central to autoimmune pathways where they produce human leukocyte antigen (HLA), adhesion and costimulatory molecules, and cytokines and chemokines [2]. The term 'autoimmune epitheliitis' has thus been suggested to describe the etiology of SS.

Systemic sclerosis (SSc) is a chronic autoimmune disease characterized by increased fibrosis and slightly enlightened pathogenesis [3, 4]. The most frequent skin sign of SSc is dermal infiltration by myofibroblasts that

(C) The Author(s). 2018 Open Access This article is distributed under the terms of the Creative Commons Attribution 4.0 International License (http://creativecommons.org/licenses/by/4.0/), which permits unrestricted use, distribution, and 
synthesize type I collagen and alpha smooth muscle actin $(\alpha-S M A)$ [4]. Increased profibrotic mediators, such as transforming growth factor (TGF) $-\beta$, and increased mammalian target of rapamycin (mTOR) activity have also been reported in dermal fibroblasts of SSc patients $[4,5]$. $\mathrm{mTOR}$ is a serine kinase that plays a role in the regulation of cell growth and proliferation. The mTOR complex includes two multiprotein complexes, mTOR complex 1 (mTORC1) and mTOR complex 2 (mTORC2) [6]. mTORC1 activates S6 kinase 1 (S6K1) and eukaryotic translation initiation factor 4E (eIF4E) which are responsible for mRNA translation [5]. mTOR also regulates cell survival and is stimulated by growth factors, nutrients, stress signals, phosphoinositol-3-kinase (PI3K), mitogenactivated protein kinase (MAPK), adenosine monophosphate (AMP), and adenosine monophosphate-activated protein kinase (AMPK). mTORC2 regulates the actin cytoskeleton and activates protein kinase $\mathrm{C}(\mathrm{PKC})-\alpha$ and Akt (protein kinase B, or PKB) [6]. mTOR multiprotein complexes have a positive effect on fibrotic interleukins (ILs). Liang et al. reported previously that IL-4, IL-6, IL-17, and TGF- $\beta$ are downregulated after mTOR inhibition with rapamycin [5]. Phosphatase and tensin homolog (PTEN) is also involved in the regulation of mTOR activity and usually inhibits mTOR via the inhibition of Akt [7]. Decreased intracellular levels of PTEN cause PI3K/ Akt/mTOR pathway activation and increase cell proliferation, survival, adhesion, migration, and angiogenesis [7]. Another mTOR regulator molecule, TGF- $\beta$, activates intracellular signaling pathways such as PI3K/Akt/mTOR and SMAD $[8,9]$. TGF- $\beta$ can also both enhance and suppress PTEN, an effect that depends on Ras/ERK pathway activation [7].

Multiple signaling pathways such as MAPK, Akt, NF- $\mathrm{kB}, \mathrm{Bcl}-2$, and JAK/STAT are found to be activated in systemic diseases in which the mTOR pathway is also an attractive therapeutic target [10]. The relationship between mTOR and increased skin fibrosis in SSc has previously been investigated both in vivo and in vitro. The role of rapamycin, an mTOR inhibitor, was investigated previously in only a murine model of SS, the results of which suggested that it has therapeutic potential [11]. We therefore aimed, in our current study, to investigate the role of the mTOR pathway in the pathologic changes observed in minor salivary gland biopsies (MSGBs) from $\mathrm{SS}, \mathrm{SSc}$, and SS/SSc overlap syndrome patients.

\section{Methods}

\section{Patients and data collection}

Patients admitted to the outpatient rheumatology clinic in our tertiary hospital between January 2007 and December 2015 were retrospectively reviewed. These patients were divided into SSc, SS, and SSc/SS overlap subgroups. Demographic (age, gender), clinical (duration of disease, presence of sicca symptoms, Schirmer's and tear breakup time (BUT) test results), and serum autoantibody data for these cases were collected using their medical records. Patients who answered positively to at least one of the questions regarding keratoconjunctivitis sicca and xerostomia were considered positive for sicca symptoms. A BUT test result $<10 \mathrm{~s}$ and a Schirmer's test finding $\leq 5 \mathrm{~mm} / 5 \mathrm{~min}$ were also considered positive indicators of sicca symptoms.

SS and SSc patients aged $\geq 18$ years who fulfilled the American-European Consensus Group (AECG) classification criteria for Sjogren's syndrome [12] and the ACR/ EULAR 2013 criteria for systemic sclerosis [13], respectively, and who underwent MSGB were screened for inclusion in the study cohort. All of the SSc patients who underwent MSGB had at least one sicca symptom or had a positive autoantibody related to sicca symptoms. We excluded patients who had received any previous treatment with mTOR inhibitors. We also excluded any secondary SS or SSc patients other than overlap cases. We did not use an informed consent form since this was a retrospective study.

\section{Histopathological evaluation}

The MSGBs were fixed with $10 \%$ buffered formalin for at least $6 \mathrm{~h}$ and then monitored with a closed-loop tissue monitoring machine overnight. Serial sections of 4-5 $\mu \mathrm{m}$ thickness were obtained from a single paraffin-embedded block for each patient, stained with hematoxylin and eosin (H\&E), and examined under a light microscope by a single expert pathologist (FC). The specimens were evaluated for the presence and number of lymphocytic foci, the presence and grade of fibrosis, and lobular or acinar atrophy. Focal lymphocytic sialadenitis with a score of $\geq 1$ foci $/ 4 \mathrm{~mm}^{2}$ was accepted as diagnostic for SS [14]. The extent of fibrosis in the MSGB specimens was assessed semiquantitatively and graded as mild (less than $25 \%$ of the surface area), moderate (between 25 and 50\%), or severe $(>50 \%)$.

\section{Immunohistochemical staining}

The MSGB specimens were evaluated by immunohistochemical (IHC) staining with antibodies for total PTEN (Spring Bioscience Rabbit Anti-Human PTEN Rabbit Monoclonal, Clone SP170), total mTOR (Spring Bioscience Rabbit Anti-Human mTOR Polyclonal Antibody), and TGF- $\beta 1$ (Spring Bioscience Rabbit Anti-Human Transforming Growth Factor $1 \beta$ Polyclonal Antibody) by a single expert pathologist (FC). IHC staining slides were evaluated via light microscopy. The positive controls used in this study were prostate adenocarcinoma tissue for PTEN, placenta for TGF- $\beta 1$, and breast cancer tissue for mTOR. 


\section{Immunohistochemical evaluation}

IHC staining of mTOR was semiquantitatively assessed as mild (1+) (Fig. 1a), moderate (2+), or strong (3+) (Fig. 1b) positivity [15]. IHC staining of PTEN was graded as negative (Fig. 1c), or as mild (1+) or strong (2+) positivity (Fig. 1d) [16]. IHC staining of TGF- $\beta 1$ was semiquantitatively assessed and graded between 0 and 4 according to the level of staining [17] (Fig. 1e, f).

\section{Statistical analysis}

Unless otherwise stated, results are presented as a mean and standard deviation (SD) or percentage as appropriate. Comparisons of categorical data between groups were made using the chi-square test. The Spearman's rank correlation test was performed for bivariate correlations between variables. All tests were two-tailed and a $P$ value of $<0.05$ was considered statistically significant for all measurements. All statistical analyses were made using the Statistical Package of Social Science (SPSS) version 16.0 software (Chicago, IL).

\section{Results}

Patient demographic and baseline features

Demographic and baseline features of the patients are presented in Table 1. Formalin-fixed MSGB sections from 58 patients with SS, 16 with SSc, and 23 with SSc/ SS overlap syndrome were initially included in the study samples. However, two female SSc samples had to be excluded to due to difficulties with IHC staining. As expected, the frequency of sicca symptoms was higher in the SS patients $(90 \%)$ than in the SSc $(77 \%)$ or overlap syndrome cases $(70 \%)$. BUT test positivity was determined as $60 \%$ in the SS patients, $36 \%$ in the SSc patients, and $74 \%$ in the overlap syndrome patients. Schirmer test positivity was found to be $58 \%$ in the SS patients, $50 \%$ in the SSc patients, and 58\% in the overlap

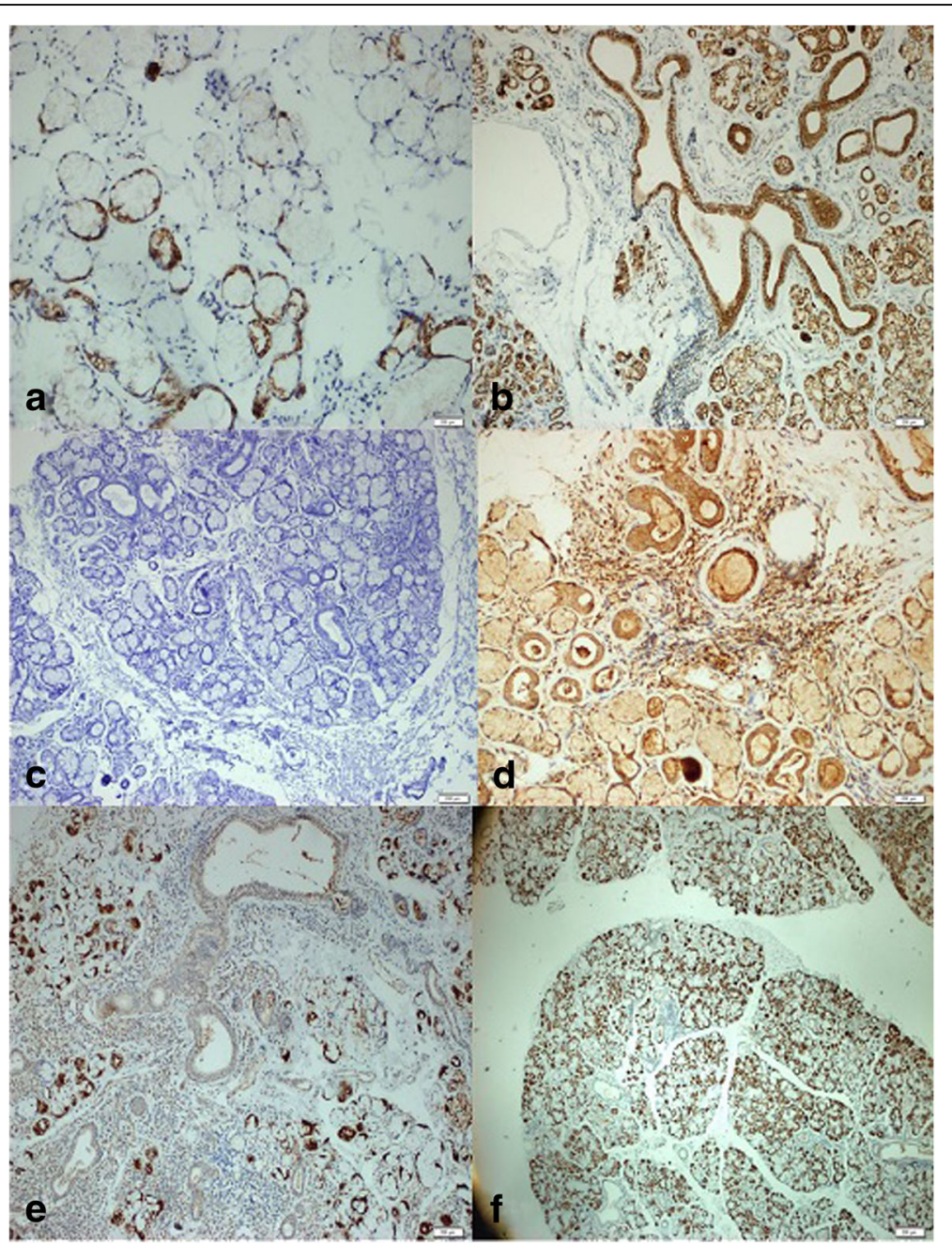

Fig. $1 \mathrm{Imm}$ unohistochemical staining of mTOR, PTEN, and TGF- $\beta 1$ in minor salivary gland biopsies. Representative samples showing mild (a) and strong (b) positivity for mTOR, negative (c) and strong positivity for PTEN (d), and mild (e) and strong positivity for TGF- $\beta$ staining (f). Positive staining for TGF- $\beta$ was mainly observed in the acinar regions of the salivary glands 
Table 1 Demographic and baseline features of the patients

\begin{tabular}{llll}
\hline & Sjogren's syndrome $(n=58)$ & Systemic sclerosis $(n=14)$ & Overlap syndrome $(n=23)$ \\
\hline Age, years (mean \pm SD) & $52.6 \pm 13.1$ & $53.9 \pm 14.2$ & $48.0 \pm 9.3$ \\
Duration of disease, months (mean \pm SD) & $58.3 \pm 23.4$ & $43.0 \pm 23.9$ & $56.8 \pm 35.4$ \\
Sicca symptom positivity, \% & 90 & 77 & 70 \\
Schirmer test positivity, \% & 58 & 50 & 58 \\
Breakup time test positivity, \% & 60 & 36 & 74 \\
Anti-Ro (SSA) or La (SSB) positivity, \% & 96 & 55 & 71 \\
\hline
\end{tabular}

syndrome patients. Anti-Ro (SSA) or anti-La (SSB) positivity was higher in the SS patients (96\%) than SSc (55\%) and overlap syndrome $(71 \%)$ patients. Examination of the biopsy specimens revealed a focus score of $\geq 1$ foci $/ 4 \mathrm{~mm}^{2}$ in 52 out of the 58 (90\%) SS patients, 22 out of the $23(96 \%)$ overlap patients, and none of the SSc patients.

\section{mTOR, PTEN, and TGF- $\beta 1$ expressions}

mTOR expression was evident in $94 \%$ of the SS group, $100 \%$ of the overlap cases, and $91 \%$ of the SSc patients (Table 2). There were no significant differences in the presence $(P=0.462)$ or degree $(P=0.622)$ of mTOR expression between these groups. PTEN protein expression was detected in $87 \%$ of the SS patients, $58 \%$ of the overlap cases, and $100 \%$ of the SSc patients (Table 2) with significant differences in the presence $(P=0.004)$ and intensity $(P=0.023)$ of staining. Although the ductal epithelial TGF- $\beta 1$ expression was similar between the groups $(P=0.345)$, acinar cell expression (Table 2) was more frequent in the SSc (73\%) and overlap patients $(86 \%)$ in comparison with the SS $(58 \%)$ cases with borderline significance $(P=0.05)$. Additionally, more of the acinar TGF- $\beta 1$ staining was strongly positive in SSC patients $(46 \%$ vs $19 \%$ and $4 \% ; P=0.004)$.

\section{Fibrosis features}

In general, fibrosis was evident in all of our patient groups but we did not observe severe fibrosis in any
MSGB sections. There was also no significant difference found between the study groups in terms of the presence of fibrosis $(P=0.833)$. Correlation analysis between the immunohistochemical staining results, presence of fibrosis, and the demographic, clinical characteristics, and autoantibodies of the SS patients showed only a negative correlation between PTEN and TGF- $\beta 1$ positivity $(r=-0.306, P=0.041)$. However, because of the small number of patients in the SSc and overlap syndrome groups, correlation analyses were not performed for these patients.

\section{Discussion}

Sicca symptoms were more frequent in our current SSc patients (77\%) than has been reported previously [18]. It should be noted, however, that all SSc patients examined in our present study had undergone MSGB as they had either sicca symptoms or autoantibody positivity suggesting an accompanying SS.

Our current findings suggest that the mTOR pathway might play an active role in the pathology of SS, SSc, and overlap syndrome. We did not find any statistically significant differences in the mTOR expression profile among our study groups indicating a common role of this pathway in these diseases. It may be appropriate, therefore, to use mTOR inhibitors more frequently in rheumatology practice.

We are not aware of any prior human study that has evaluated mTOR inhibitors in SS patients. Shah

Table 2 Immunohistochemical staining results in the three patient groups (mTOR, PTEN, and TGF- $\beta 1$ in acinus)

\begin{tabular}{|c|c|c|c|c|c|}
\hline & & Negative & Mild positivity & Moderate positivity & Strong positivity \\
\hline \multirow[t]{3}{*}{ mTOR } & SS & $3(6)$ & $25(46)$ & $20(37)$ & $6(11)$ \\
\hline & Overlap syndrome & 0 & $11(55)$ & $9(45)$ & 0 \\
\hline & SSC & $1(9)$ & $4(36)$ & $5(46)$ & $1(9)$ \\
\hline \multirow[t]{3}{*}{ PTEN } & SS & $6(13)$ & $23(49)$ & - & $18(38)$ \\
\hline & Overlap syndrome & $8(42)$ & $5(26)$ & - & $6(32)$ \\
\hline & SSC & 0 & $7(58)$ & - & $5(42)$ \\
\hline \multirow[t]{3}{*}{ TGF- $\beta 1$} & SS & $23(42)$ & $20(36)$ & $10(18)$ & $2(4)$ \\
\hline & Overlap syndrome & $3(14)$ & $10(48)$ & $4(19)$ & $4(19)$ \\
\hline & SSC & $3(27)$ & $2(18)$ & $1(9)$ & $5(46)$ \\
\hline
\end{tabular}

All values are shown as $n$ (\%)

mTOR mammalian target of rapamycin, PTEN phosphatase and tensin homolog, SS Sjogren's syndrome, SSC systemic sclerosis, TGF transforming growth factor 
and colleagues have previously reported in a mouse model of SS that the mTOR inhibitor sirolimus may suppress the lymphocytic infiltration of lacrimal glands [11]. Our present observations may encourage other investigators to test mTOR inhibitors as potential new SS therapeutics.

Forestier et al. recently showed an altered B cell homeostasis in SSc patients compared with healthy controls [19]. This altered B cell homeostasis was found to be related to the mTOR pathway. However, the most important limitation of this study is the lack of a control group. Thus, further studies with healthy control groups may shed light on the pathogenesis of SSc and SS and may help us to understand the role of the mTOR pathway in the rheumatologic diseases.

We found a statistically significant difference between the acinar TGF- $\beta 1$ expression levels in our study groups. As expected, strong TGF- $\beta 1$ expression, a well-known fibrosis indicator, was found most frequently in the SSc patients. In addition, PTEN expression was similar in SS and SSc patients and this was significantly different from the overlap patients. These data suggest that epithelopathogenesis follows a different pathway in overlap syndrome than in SS or SSc. Although PTEN is known as an endogenous mTOR inhibitor, it has also previously been shown that increased mTOR activity is accompanied by increased PTEN levels and it was hypothesized that this might be due an autoimmune-related impairment in the PTEN pathway [7]. However, further studies are needed to investigate the effects of PTEN and PTEN-related molecules on autoimmune diseases.

Our correlation analysis in the SS group did not reveal any association between mTOR, PTEN, or TGF- $\beta 1$ expression. This might indicate that PTEN and TGF- $\beta 1$ operate independently of the mTOR pathway in SS pathogenesis. We also found a negative association between TGF- $\beta 1$ and PTEN expression in our SS samples. Under normal physiological conditions in the cell, TGF- $\beta 1$ may have both enhancing and reducing effects on PTEN. When the Ras/ERK pathway is activated, TGF- $\beta$ suppresses PTEN by the SMAD4-independent signal pathway [7]. However, when the Ras/ERK pathway is inactivated, TGF- $\beta$ upregulates the classic SMAD-dependent PTEN molecule [7]. The negative correlation between TGF- $\beta 1$ and PTEN in SS suggests that the Ras/ERK pathway is active in this disease, but no precise information is yet available because other pathway members have yet to be identified. In addition, some notable limitations of our present study include the small number of SSc and overlap syndrome patients, the lack of sicca controls, and other reasons and technical difficulties with analyzing some of the biopsy materials.

\section{Conclusions}

In conclusion, mTOR may be one of the common pathways leading to the pathology/inflammation observed in both SS and SSc and may provide a new alternative for the development of new treatments for both diseases. Additionally, higher PTEN and TGF- $\beta 1$ expression, in particular a higher acinar TGF- $\beta 1$ level, may be a distinctive feature of SSc.

\section{Key messages}

- The mTOR pathway appears to be similarly active in minor salivary gland biopsies of SS and SSc patients.

- PTEN and TGF- $\beta 1$ expression may be a distinctive feature of salivary gland pathology in SSc.

\begin{abstract}
Abbreviations
a-SMA: Alpha smooth muscle actin; ACR: American College of Rheumatology; AECG: American-European Consensus Group; Akt: Protein kinase B; AMP: Adenosine monophosphate; AMPK: Adenosine monophosphate-activated protein kinase; BCl2: B-cell lymphoma 2; BUT: Breakup time; CD4: Cluster of differentiation; elF4E: Eukaryotic translation initiation factor 4E; ERK: Extracellular signalregulated kinases; EULAR: European League Against Rheumatism; H\&E: Hematoxylin and eosin; HLA: Human leukocyte antigen; HHC: Immunohistochemical; IL: Interleukin; JAK: Janus activated kinase; MAPK: Mitogen-activated protein kinase; mRNA: Messenger ribonucleic acid; MSGB: Minor salivary gland biopsy; mTOR: Mammalian target of rapamycin; mTORC1: Mammalian target of rapamycin complex 1; mTORC2: Mammalian target of rapamycin complex 2; NF-KB: Nuclear factor kappa B; PI3K: Phosphoinositol-3-kinase; PKB: Protein kinase B; PKC: Protein kinase C; PTEN: Phosphatase and tensin homolog; S6K1: S6 kinase 1; SD: Standard deviation; SMAD: Small mothers against decapentaplegic; SPSS: Statistical Package of Social Science; SS: Sjogren's syndrome; SSc: Systemic sclerosis; STAT: Signal transducer and activator of transcription; TGF: Transforming growth factor
\end{abstract}

\section{Funding}

This study was supported by the Izmir Katip Celebi University Science Research Project Coordinator.

\section{Availability of data and materials}

The datasets used and/or analyzed during the current study are available from the corresponding author on reasonable request.

\section{Authors' contributions}

SA designed the research and revised the manuscript. ZS analyzed data and wrote the manuscript. ZZG collected data, analyzed data, and wrote the manuscript. FÇ helped with pathology diagnosis. MÖ and DS participated in case and data collection. SG and ÖG helped optimize the research and proofread the paper. All authors read and approved the final manuscript.

\section{Ethics approval and consent to participate}

Our study complies with the declaration of Helsinki and was approved by the locally appointed ethics committee (The ethics committee of Izmir Katip Celebi University).

\section{Consent for publication}

We did not use an informed consent form since this was a retrospective study.

\section{Competing interests}

The authors declare that they have no competing interests.

\section{Publisher's Note}

Springer Nature remains neutral with regard to jurisdictional claims in published maps and institutional affiliations. 


\section{Author details}

'Department of Internal Medicine, Division of Nephrology, Izmir Katip Celebi University School of Medicine, Karabağlar, 35360 İmir, Turkey. ${ }^{2}$ Dogubeyazit Public Hospital, Internal Medicine, Agri, Turkey. ${ }^{3}$ Department of Pathology, Izmir Katip Celebi University, Izmir, Turkey. ${ }^{4}$ Department of Internal Medicine, Division of Rheumatology, Izmir Katip Celebi University, Izmir, Turkey.

Received: 27 April 2018 Accepted: 6 July 2018

Published online: 04 August 2018

\section{References}

1. Kassan SS, Moutsopoulos HM. Clinical manifestations and early diagnosis of Sjögren syndrome. Arch Intern Med. 2004;164(12):1275-84. https://doi.org/ 10.1001/archinte.164.12.1275

2. Moriyama M, Tanaka A, Maehara T, Furukawa S, Nakashima H, Nakamura S. T helper subsets in Sjögren's syndrome and lgG4-related dacryoadenitis and sialoadenitis: a critical review. J Autoimmun. 2014; https://doi.org/10.1016/j. jaut.2013.07.007.

3. Manno R, Boin F. Immunotherapy of systemic sclerosis. Immunotherapy. 2010;2(6):863-78. https://doi.org/10.2217/imt.10.69.

4. Gilbane AJ, Denton CP, Holmes AM. Scleroderma pathogenesis: a pivotal role for fibroblasts as effector cells. Arthritis Res Ther. 2013;15(3):215. https:// doi.org/10.1186/ar4230.

5. Liang M, Lv J, Chu H, Wang J, Chen $X$, Zhu X, et al. Vertical inhibition of $\mathrm{PI3K} / \mathrm{Akt} / \mathrm{mTOR}$ signaling demonstrates in vitro and in vivo anti-fibrotic activity. J Dermatol Sci. 2014;76(2):104-11. https://doi.org/10.1016/j.jdermsci. 2014.08.002.

6. Populo H, Lopes JM, Soares P. The mTOR signalling pathway in human cancer. Int J Mol Sci. 2012;13(2):1886-918. https://doi.org/10.3390/ ijms13021886.

7. Assinder SJ, Dong Q, Kovacevic Z, Richardson DR. The TGF-beta, PI3K/Akt and PTEN pathways: established and proposed biochemical integration in prostate cancer. Biochem J. 2009;417(2):411-21. https://doi.org/10.1042/ BJ20081610.

8. Xu J, Lamouille S, Derynck R. TGF-beta-induced epithelial to mesenchymal transition. Cell Res. 2009;19(2):156-72. https://doi.org/10.1038/cr.2009.

9. Lamouille S, Connolly E, Smyth JW, Akhurst RJ, Derynck R. TGF-ß-induced activation of mTOR complex 2 drives epithelial-mesenchymal transition and cell invasion. J Cell Sci. 2012; https://doi.org/10.1242/jcs.095299.

10. Wu T, Mohan C. The AKT axis as a therapeutic target in autoimmune diseases. Endocrine, Metab immune Disord -drug Targets. 2009;9(2):145-50.

11. Shah M, Edman MC, Janga SR, Shi P, Dhandhukia J, Liu S, et al. A rapamycin-binding protein polymer nanoparticle shows potent therapeutic activity in suppressing autoimmune dacryoadenitis in a mouse model of Sjogren's syndrome. J Control Release. 2013;171(3):269-79. https://doi.org/ 10.1016/j.jconrel.2013.07.016.

12. Vitali C, Bombardieri S, Jonsson R, Moutsopoulos HM, Alexander EL, Carsons SE, et al. Classification criteria for Sjögren's syndrome: a revised version of the European criteria proposed by the American-European consensus group. Ann Rheum Dis. 2002;61(6):554-8.

13. van den Hoogen F, Khanna D, Fransen J, Johnson SR, Baron M, Tyndall A, et al. 2013 classification criteria for systemic sclerosis: an American College of Rheumatology/European league against rheumatism collaborative initiative. Ann Rheum Dis. 2013;72(11):1747-55. https://doi.org/10.1136/annrheumdis2013-204424.

14. Chisholm DM, Mason DK. Labial salivary gland biopsy in Sjögren's disease. J Clin Pathol. 1968;21(5):656-60.

15. Mutee AF, Kaur G, Kumar G, Muhammad TST, Khalid IA, Tan ML Immunohistochemical evaluation of mTOR and Beclin-1 protein expression in human breast cancer and adjacent normal tissues, a study in Malaysian patients. Open Pathol J. 2009;3:111-7. https://doi.org/10.2174/ 1874375700903010111.

16. Sakr RA, Barbashina V, Morrogh M, Chandarlapaty S, Andrade VP, Arroyo CD et al. Protocol for PTEN expression by immunohistochemistry in formalinfixed paraffin-embedded human breast carcinoma. Appl Immunohistochem Mol Morphol. 2010;18(4):371-4. https://doi.org/10.1097/PAl. Ob013e3181d50bd5.

17. Xie $S$, Macedo P, Hew M, Nassenstein C, Lee KY, Chung KF. Expression of transforming growth factor- $\beta$ in idiopathic cough. Respir Res 2009; doi: https://doi.org/10.1186/1465-9921-10-40.
18. Hunzelmann N, Genth E, Krieg T, Lehmacher W, Malchers I, Meurer M, et al. The registry of the German network for systemic scleroderma: frequency of disease subsets and patterns of organ involvement. Rheumatology (Oxford). 2008;47(8):1185-92. https://doi.org/10.1093/rheumatology/ken179.

19. Forestier A, Guerrier T, Jouvray M, Giovannelli J, Lefèvre G, Sobanski V, et al. Altered B lymphocyte homeostasis and functions in systemic sclerosis. Autoimmun Rev. 2018;17(3):244-55. https://doi.org/10.1016/j.autrev.2017.10.015.

\section{Ready to submit your research? Choose BMC and benefit from:}

- fast, convenient online submission

- thorough peer review by experienced researchers in your field

- rapid publication on acceptance

- support for research data, including large and complex data types

- gold Open Access which fosters wider collaboration and increased citations

- maximum visibility for your research: over $100 \mathrm{M}$ website views per year

At $\mathrm{BMC}$, research is always in progress.

Learn more biomedcentral.com/submissions 